This article is licensed under the Creative Commons Attribution-NonCommercial 4.0 International License (CC BY-NC) (http://www.karger.com/Services/OpenAccessLicense). Usage and distribution for commercial purposes requires written permission.

\title{
Plexiform Neurofibroma Involving the Lacrimal Gland
}

\author{
Mikael Hofsli $^{\mathrm{a}} \quad$ Nico Gampenrieder ${ }^{\mathrm{b}} \quad$ Steffen Heegaard $^{\mathrm{c}}$ \\ ${ }^{a}$ Department of Ophthalmology, Naestved Hospital, Naestved, Denmark; ${ }^{b}$ Department of \\ Ophthalmology, Odense University Hospital, Odense, Denmark; ' Department of \\ Ophthalmology and Department of Pathology, Eye Pathology Section, \\ Righospitalet-Glostrup Hospital, University of Copenhagen, Copenhagen, Denmark
}

\section{Keywords}

Plexiform neurofibroma $\cdot$ Lacrimal gland $\cdot$ Neurofibromatosis type $1 \cdot$ Salivary gland

\begin{abstract}
Background: To present a rare case of a 2-year-old girl with neurofibromatosis type 1 (NF1) who presented with ptosis of the right upper eyelid along with a tumor in the eyelid. Methods: A magnetic resonance imaging scan of the orbit revealed a solid tumor located extraconally at the site of the right lacrimal gland. A transcranial orbitotomy was performed. Results: Histopathological examination demonstrated expanded nerve branches/fascicles cut in various planes in between normal lacrimal gland acini. These findings were consistent with a plexiform neurofibroma presumably deriving from the lacrimal nerve and/or a supraorbital nerve branch. Conclusion: This is the first case of a plexiform neurofibroma involving the lacrimal gland ever described and the tumor shows similarities with neurofibroma in other salivary glands with a high recurrence rate. Plexiform neurofibromas are frequently seen in patients with NF1 and rarely undergo malignant transformation.
\end{abstract}




\section{Case Reports in Ophthalmology}

\section{Introduction}

Neurofibromatosis type 1 (NF1) has a prevalence of 1:2,500 to 1:3,500 people, regardless of ethnic and racial background and is inherited in an autosomal dominant manner that exhibits complete penetrance [1]. Half of the cases are a result of a sporadic gene mutation [1]. The NF1 gene is located at the long arm of chromosome 17 [1]. Inactivating mutations of NF1 cause a decrease in the amount of the gene product, neurofibromin, and lead to increased intracellular signaling through Ras [1].

The National Institutes of Health (NIH) [2] developed in 1987 the diagnostic criteria for NF1, and updated them in 1997 [3]. An individual meets the criteria of having NF1 and should be diagnosed if 2 or more specific features are present (Table 1) [2]. The diagnosis of NF1 can in most cases be made based on these criteria; in rare cases, it is necessary to pursue genetic testing [1].

Peripheral nerve sheath tumors (PNST) often involve the neck and head region, but are uncommon in the orbit [4]. Plexiform neurofibromas, in contrast to other PNST, are diagnosed in early life between the age of 1 and 5 years [4]. Plexiform neurofibromas of the salivary gland are rare, but when present, they are commonly located in the parotid gland [5]. They are locally invasive tumors and not prone to metastasizing [6].

This case is the only reported case of a plexiform neurofibroma involving the lacrimal gland.

\section{Materials and Methodology}

A 2-year-old girl presented to the clinic with ptosis of the right upper eyelid that had persisted since the age of 3-4 months. The girl was first seen by an ophthalmologist at the age of 1 year due to ptosis of the right eyelid. The pupil was free of the right-sided ptosis, but the ptosis progressed. The patient had a twin sister without symptoms and no other family disposition to ptosis. At the time of referral (December 1998) NF1 was suspected because of the presence of café-au-lait spots on the skin of the abdomen and thorax.

The initial clinical examination revealed no signs of strabismus, and the motility of both eyes was normal. There was significant ptosis of the right upper eyelid covering most of the cornea, but the patient was able to elevate the eyelid $3 \mathrm{~mm}$ over the upper edge of the pupil. There was a characteristic s-shaped deformity of the right upper eyelid typical of NF1, and the eyelid was soft on palpation (Fig. 1A). The left eye was normal. Ophthalmoscopy was performed at a later consultation and was normal in both eyes. Magnetic resonance imaging (MRI) scans of the cerebrum and the orbit were performed and showed exophthalmus and a solid tumor, $1 \mathrm{~cm}$ at the largest axis, located extraconally at the site of the lacrimal gland and highly suggestive of a plexiform neurofibroma or a lacrimal gland tumor (Fig. 1B). No sign of optic pathway glioma, infiltration of the eye or the bone was found.

In June 1999, the tumor was excised in toto by transcranial orbitotomy, and orbital roof reconstruction was performed in the same operation 6 months after the first consultation. On clinical examination 2 months after surgery, the ptosis was reduced to a mild/moderate type. The shape of the eyelid returned to normal. The right-sided ptosis was measured to 1$2 \mathrm{~mm}$, with a closing defect of $1 \mathrm{~mm}$. Fourteen months after surgery, there was no change in 


\section{Case Reports in Ophthalmology}

Case Rep Ophthalmol 2017;8:67-72

DOI: $10.1159 / 000456067$

(C) 2017 The Author(s). Published by S. Karger AG, Basel www.karger.com/cop

Hofsli et al.: Plexiform Neurofibroma Involving the Lacrimal Gland

the patient's ptosis compared with 2 months after surgery; besides, the closing defect had disappeared. Visual acuity (VA) was 0.25 bilaterally.

In October 2005, the patient was again referred to the clinic on suspicion of tumor recurrence. Clinical examination revealed a $3 \times 1 \mathrm{~cm}$ freely movable tumor located subcutaneous at the lateral cantus. Based on the clinical examination, no orbital involvement was suspected. The right eye was slightly downward displaced, and there was a $5^{\circ}$ exotropia but normal motility. VA was 0.12 in the right eye and 0.66 in the left eye. Tear production was zero in the right eye. Levator function was $7 / 17 \mathrm{~mm}$. Because of compromised tear production, the patient received punctum plugs. It was decided to remove the tumor, and during surgery it was clear that the tumor consisted of 2 tumors $(2 \times 2$ and $0.5 \times 0.5 \mathrm{~cm})$ located in the eyelid at the lateral side of the right eye. Both tumors were removed and histopathological examination revealed recurrence of plexiform neurofibroma.

The patient underwent ptosis surgery in November 2010, and in August 2013 re-ptosis surgery was performed with removal of fibroadenomatous tissue in the eyelid of the right eye.

\section{Results}

\section{Histopathological Examination}

The specimen from the lacrimal gland measured $20 \times 14 \times 14 \mathrm{~mm}$. Microscopical examination of the initial tumor showed nerve sheath proliferation confined within the perineurium of multiple disorganized nerve fascicles. The nerve fascicles intermingled between the normal lacrimal acini. The nerve fibers were arranged in a concentric manner with areas of myxoid changes. The tumor was characterized by tortuous proliferation of all components of peripheral nerves including axons, Schwann cells, fibroblasts, and perineurial cells. The amount of normal lacrimal tissue constituted 10-15\% (Fig. 1C, D).

Immunohistochemically, the tumor cells stained positive for epithelial membrane antigen (perineurium), neurofilament (residual axons), neuron-specific enolase, S-100 (Schwann cells), CD34 (admixed fibroblasts), and vimentin. Tumor cells stained negative for pancytokeratin (CKAE-CAM) and Mib-1 (KI-67). The histological features were consistent with a plexiform neurofibroma presumably deriving from the lacrimal nerve and/or a supraorbital nerve branch.

\section{Discussion}

The most common tumor found in the lacrimal gland is pleomorphic adenoma, but a spectrum of lymphoid tumors may also develop [7]. In a Danish study, a total of 232 lesions were characterized [8]. Approximately half of the lesions were neoplasms, and half of these were malignant [8].

PNST can involve the nerve fascicles or cranial branches II-VII in the orbit [4]. Plexiform neurofibromas in NF1 often present in childhood or adolescence with a characteristic s-shaped curvature and swelling of the upper eyelid [4]. Orbitotemporal neurofibromatosis (OTNF) is a condition where patients develop grossly disfiguring neurofibromas of the orbit, 


\section{Case Reports in Ophthalmology}

\begin{tabular}{l|l}
\hline Case Rep Ophthalmol 2017:8:67-72 \\
\hline DOI: 10.1159/000456067 & $\begin{array}{l}\text { (c) } 2017 \text { The Author(s). Published by S. Karger AG, Basel } \\
\text { www.karger.com/cop }\end{array}$
\end{tabular}

Hofsli et al.: Plexiform Neurofibroma Involving the Lacrimal Gland

temporal and facial region with onset in childhood [9]. Our patient had lacrimal gland and eyelid involvement, without absence or involvement of the sphenoid bone which is a characteristic skeletal abnormality in OTNF [9].

Neurofibromas constitute $0.4 \%$ of all salivary neoplasms [10]. Plexiform neurofibromas are slow-growing, locally infiltrating benign tumors [10]. They arise from diffuse enlargement of multiple nerve fascicles and its branches, resulting in nerve thickening [10]. Due to their locally invasive nature, they can cause cosmetic and functional deformities in the head and neck region [6].

Plexiform neurofibromas are common in patients with NF1 (30-50\%), but only in 5$10 \%$ of cases does the tumor undergo malignant transformation [1]. Plexiform neurofibromas of the salivary glands are most commonly reported in the parotid gland $[5,11,12]$. Submandibular salivary gland neurofibromas are rare [11]. There has been 1 reported case of an isolated neurofibrosarcoma of the lacrimal gland in the literature [13]. This is the first described plexiform neurofibroma involving the lacrimal gland.

In the management of orbitotemporal NF1, no firm guidelines are currently established [4]. Children and adolescents typically experience rapid growth of neurofibromatosis and should remain under close observation, monitoring their visual development to allow timely intervention with appropriate surgical procedures [4, 14]. The risk of developing amblyopia in children should always be taken into consideration [4]. In our case, the patient developed amblyopia, probably because of severe ptosis for several years, leading to a compromised VA of the right eye. All treatments need to be individualized and should ideally be managed through a multidisciplinary medical approach [4, 14].

The treatment of choice for benign neurofibromas is complete excision with intact tumor capsule [4]. Lateral orbitotomy is commonly used when the tumor is located superolaterally [4]. Plexiform neurofibromas often present with diffuse soft tissue infiltration, which makes complete resection difficult [9]. Only the rare, well-circumscribed neurofibromas can be removed intact through an anterolateral orbitotomy [9]. Debulking surgery for plexiform neurofibromas should only be performed in special cases, due to the tendency to recur when only partly removed [4]. Our patient initially underwent transcranial orbitotomy, as the operation was performed by neurosurgeons. Multiple tumor recurrences developed in the present case.

In summary, neurofibroma involving the lacrimal gland is extremely rare, and shows similarities with lesions in other salivary glands. This is the first reported case of a plexiform neurofibroma involving the lacrimal gland. Plexiform neurofibromas are frequently seen in patients with NF1.

\section{Acknowledgements}

The authors of this article and their institution have not received any financial support or services in connection with the submitted work. The authors had full access to all of the data in the study and take responsibility for the integrity of the data and the accuracy of the data analysis. 


\section{Statement of Ethics}

The authors have no ethical conflicts to disclose.

\section{Disclosure Statement}

There was no conflict of interest.

\section{References}

1 Anderson JL, Gutmann DH: Neurofibromatosis type 1. Handb Clin Neurol 2015;132:75-86.

-2 National Institutes of Health Consensus Development Conference. Neurofibromatosis. Conference statement. Arch Neurol 1988;45:575-578.

3 Gutmann DH, Aylsworth A, Carey JC, Korf B, Marks J, Pyeritz RE, et al: The diagnostic evaluation and multidisciplinary management of neurofibromatosis 1 and neurofibromatosis 2. JAMA 1997;278:5157.

4 Sweeney AR, Gupta D, Keene CD, Cimino PJ, Chambers CB, Chang S-H, et al: Orbital peripheral nerve sheath tumors. Surv Ophthalmol 2017;62:43-57.

5 Guraya SS, Prayson RA: Peripheral nerve sheath tumors arising in salivary glands: a clinicopathologic study. Ann Diagn Pathol 2016;23:38-42.

-6 Dereköy S, Sefali M: Plexiform neurofibroma of the submandibular gland. J Laryngol Otol 2000;114:643-645.

7 Won SJ, Kook JA, Mi RP, Ji YK, Jae JC, Bum SK, et al: The radiological spectrum of orbital pathologies that involve the lacrimal gland and the lacrimal fossa. Korean J Radiol 2007;8:336-342.

-8 Von Holstein SL, Therkildsen MH, Prause JU, Stenman G, Siersma VD, Heegaard S: Lacrimal gland lesions in Denmark between 1974 and 2007. Acta Ophthalmol 2013;91:349-354.

-9 Erb MH, Uzcategui N, See RF, Burnstine MA: Orbitotemporal neurofibromatosis: classification and treatment. Orbit 2007;26:223-228.

10 Tati SY, Gole GN, Prabhala S, Gole SG: Plexiform neurofibroma of the submandibular salivary gland: a rare tumour. Indian J Surg 2011;73:224-226.

11 Shekar T, Gole G, Prabhala S, Gole S: Plexiform neurofibroma: a rare tumor of submandibular salivary gland. J Surg Tech Case Rep 2010;2:81.

-12 Souaid J-P, Nguyen V-H, Zeitouni AG, Manoukian J: Intraparotid facial nerve solitary plexiform neurofibroma: a first paediatric case report. Int J Pediatr Otorhinolaryngol 2003;67:1113-1115.

13 Pattanayak SP, Mathur JS, Thakur V, Khanna S: Neurofibrosarcoma of lacrimal gland. Indian J Ophthalmol 1987;35:44-48.

14 Lee V, Ragge NK, Collin JRO: Orbitotemporal neurofibromatosis. Clinical features and surgical management. Ophthalmology 2004;111:382-388. 


\section{Case Reports in Ophthalmology} www.karger.com/cop

Hofsli et al.: Plexiform Neurofibroma Involving the Lacrimal Gland
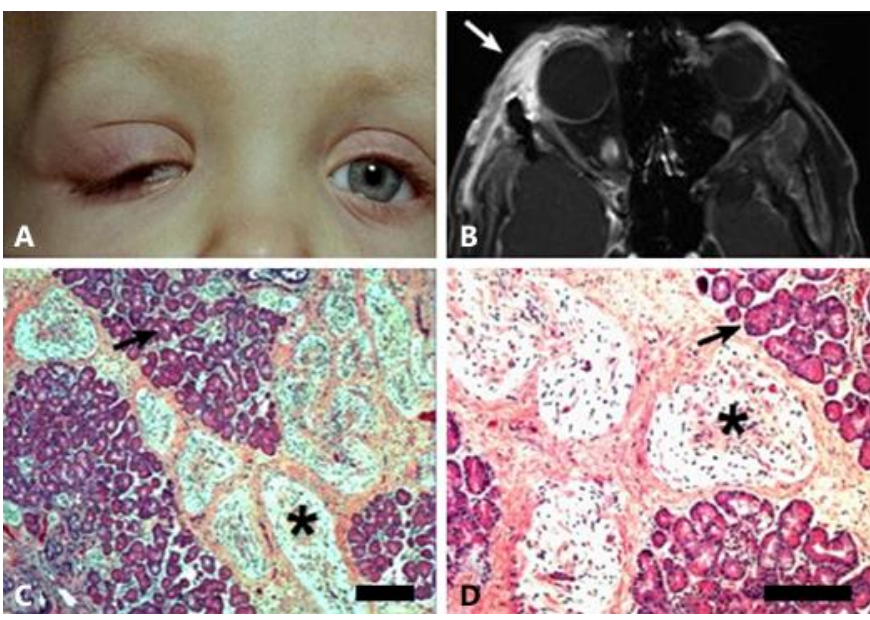

Fig. 1. A Initial clinical photograph of the patient (2 years of age) showing ptosis of the right eyelid and a solid mass located on the right upper lid. B Magnetic resonance imaging scan of the cerebrum and orbit revealing a solid tumor located extraconally at the site of the right lacrimal gland (arrow). C Markedly enlarged nerve segments and thickened nerve bundles $\left({ }^{*}\right)$ within the lacrimal gland tissue (arrow). Hematoxylin and eosin section. Scale bar $=1: 200 \mu \mathrm{m}$. D Higher magnification showing bundles of nerve fibers arranged in a concentric manner with areas of myxoid changes. Schwann cells and fibroblasts are seen. Hematoxylin and eosin section. Scale bar $=1: 200 \mu \mathrm{m}$. Normal lacrimal gland tissue (arrow) is seen between the nerve fiber bundles $(*)$.

Table 1. The diagnostic criteria for NF1 developed by the National Institutes of Health (NIH) in 1987 [2]

1. Six or more café-au-lait spots $>5 \mathrm{~mm}$ in greatest diameter in prepubertal individuals; and $>15 \mathrm{~mm}$ in greatest diameter in postpubertal individuals

2. Two or more neurofibromas of any type or 1 plexiform neurofibroma

3. Freckling in the axillary or inguinal regions

4. Optic glioma

5. Two or more Lisch nodules (iris harmartomas)

6. A distinctive osseous lesion such as sphenoid dysplasia or thinning of long bone cortex with or without pseudoarthrosis

7. A first-degree relative (parent, sibling, or offspring) with neurofibromatosis by the above criteria

The criteria are met in an individual if two or more of the features listed are present. 